Mathematical Modelling and Analysis

Volume 20 Number 3, May 2015, 329-345

http://dx.doi.org/10.3846/13926292.2015.1048757

(c) Vilnius Gediminas Technical University, 2015
Publisher: Taylor\&Francis and VGTU

http://www.tandfonline.com/TMMA

ISSN: $1392-6292$

eISSN: 1648-3510

\title{
Generating Pareto Optimal Solutions of Multi-Objective LFPP with Interval Coefficients Using $\epsilon$-Constraint Method
}

\author{
Suvasis Nayak and Akshay Ojha \\ School of Basic Science, Indian Institute of Technology Bhubaneswar \\ India \\ E-mail: nksuvasis@gmail.com \\ E-mail(corresp.): akojha57@yahoo.com
}

Received June 3, 2014; revised May 1, 2015; published online May 15, 2015

\begin{abstract}
This paper illustrates a procedure to generate pareto optimal solutions of multi-objective linear fractional programming problem (MOLFPP) with closed interval coefficients of decision variables both in objective and constraint functions. $\epsilon$-constraint method is applied to produce pareto optimal solutions comprising most preferred solution to satisfy all objectives. A numerical example is solved using our proposed method and the result so obtained is compared with that of fuzzy programming which justifies the efficiency and authenticity of the proposed method.
\end{abstract}

Keywords: multi-objective LFPP, $\epsilon$-constraint method, closed interval coefficients, Fuzzy programming.

AMS Subject Classification: 90C32.

\section{Introduction}

Mathematical optimization is described as the science of determining the best solution to the mathematically modelled real world problems. Generally, it is the process of formulation and solution of constrained or unconstrained optimization problems occurring in every branch of science and engineering designed problems. Various real world problems concerned with decision making situations can be modelled as constrained or unconstrained multi-objective optimization (i.e., vector optimization or pareto optimization) problems. In multi-objective optimization problems, usually there does not exist single solution which satisfies all the objectives, however a set of pareto optimal(noninferior, non-dominated) solutions can be generated from which the decision maker (DM) has a choice to decide the most preferred pareto optimal solution. In the recent past, researchers have given more emphasis on multiobjective techniques to handel fractional optimization problems. In fractional programming problems, the numerators and denominators of the objectives or 
the constraints are considered as linear or nonlinear functions. In order to optimize a multi-objective fractional programming problem, several ratios of functions need to be optimized simultaneously. It has wide range of application in numerous important fields like finance, engineering, business, economics, management, and so forth. Various mathematical optimization problems comprising fractional objectives are frequently encountered $[1,20]$ in many real life situations like optimization of profit/cost, output/employee, cost/time, inventory/sale, etc. Linear fractional programming problem (LFPP) contains some linear constraints and a fractional objective function i.e., fraction of two linear functions was developed by Hungarian mathematician B.Martos [14] in 1960. Several methods have been proposed in literature for obtaining the optimal solution of LFPP. Charnes and Cooper [5] developed a variable transformation technique in 1960 which converts the fractional objective function to a nonfractional linear form with an additional variable and a constraint. Costa [7] proposed weighted sum technique to solve linear fractional programming problem. Jain and Arya [12] proposed an inverse optimization model to find out the solutions of a linear fractional programming problem. Borza et al. [3] derived a method to solve LFPP with interval coefficients in the objective function. Effati and Pakdaman [8] converted the objective function with interval coefficients to an interval valued objective function. Some important concepts on interval coefficients in the constraints and the objective functions are explained by H.C. Wu. [22]. Multi-objective LFPP comprises many fractional objective functions to be optimized subject to a certain set of linear constraints. Many methods have been proposed for solution in this regard. Pal and Sen [19] proposed goal programming method to solve interval valued MOLFPP. Milan [11] discussed linear fractional programming problem under interval uncertainty to obtain a range for optimal values of the objective functions. Nykowski and Zolkiewski [17] proposed a compromise procedure to determine the nondominated solution whereas Caballero and Hernandez [4] developed a method to find a set of weakly efficient solutions of MOLFPP. Suprajitno [21] proposed a method to solve multiobjective linear programming with interval coefficients. Bellman and Zadeh [2] presented some basic concepts regarding fuzzy programming which is widely followed. Luhandjula [13] proposed fuzzy approach to find the efficient solution of MOLFPP.

Many real world problems when are mathematically modelled, do not carry certain values as coefficients. In such situations, intervals of fixed values are better to be assumed as the coefficients of the decision variables. In this paper, closed intervals of certain values are assumed as the coefficients of the decision variables both in objective and constraint functions of a multi-objective LFPP. Relative minimum and maximum values of each objective function with respect to others are determined then $\epsilon$-constraint method due to Haimes et al. [10] is used to derive a set of non-inferior solutions from which the decision maker(DM) decides the most preferred optimal solution to satisfy all the objectives with best compromising objective values on preference basis. Efficiency of our proposed method is ensured by comparing the results with the existing fuzzy programming(Zimmermann's max-min operator) method through an example. The organisation of the paper is as follows: Following introduction, some useful 
results regarding interval arithmetic have been presented in Section 2. Some brief ideas of multi-objective optimization problem and existence of it's optimal solution have been concisely interpreted in Section 3 whereas $\epsilon$-constraint method has been incorporated in Section 4. Fuzzy programming method to solve a multi-objective minimization problem has been explained in Section 5 . Our proposed method for solving multi-objective linear fractional programming problem with interval coefficients has been discussed in Section 6. An illustrative example has been solved using both the proposed method and existing fuzzy programming method separately then some remarks regarding the solutions obtained are added in Section 7. Finally, some conclusions are drawn from the discussion have been incorporated in Section 8.

\section{Preliminaries of Interval Arithmetic}

Assume that I denotes the colletion of all closed intervals in real line $\mathbb{R}$. Let $b, c \in \mathrm{I}$ such that $b=\left[b^{L}, b^{U}\right], c=\left[c^{L}, c^{U}\right]$, where $b^{L}, c^{L}, b^{U}, c^{U} \in \mathbb{R}$ are lower and upper bounds of $b$ and $c$. The results of interval arithmetic given by [16] are interpreted as follows.

- $b+c=\left[b^{L}+c^{L}, b^{U}+c^{U}\right]$.

- for $\alpha \in \mathbb{R}, \alpha b= \begin{cases}{\left[\alpha b^{L}, \alpha b^{U}\right],} & \alpha \geq 0, \\ {\left[\alpha b^{U}, \alpha b^{L}\right],} & \alpha<0 .\end{cases}$

- $b-c=\left[b^{L}-c^{U}, b^{U}-c^{L}\right]$.

- $b c=\left[b^{L}, b^{U}\right]\left[c^{L}, c^{U}\right]=\left[\operatorname{Min} D_{1}, \operatorname{Max} D_{1}\right]$, where $D_{1}=\left\{b^{L} c^{L}, b^{L} c^{U}, b^{U} c^{L}, b^{U} c^{U}\right\}$.

- $b / c=\left[b^{L}, b^{U}\right] /\left[c^{L}, c^{U}\right]=\left[\operatorname{Min} D_{2}, \operatorname{Max} D_{2}\right]$, where $D_{2}=\left[\frac{b^{L}}{c^{L}}, \frac{b^{L}}{c^{U}}, \frac{b^{U}}{c^{L}}, \frac{b^{U}}{c^{U}}\right]$ and $0 \notin c$.

\section{Multi-Objective Optimization Problem (MOOP)}

Optimization is an important activity in many fields of science and engineering. The classical frame work for optimization is to find optimal value of objective functions with respect to given constraints. A multi-objective optimization problem $[9,15]$ can be stated as:

$$
\begin{aligned}
& \max / \min f(x)=\left(f_{1}(x), f_{2}(x), \ldots, f_{k}(x)\right) \\
& \text { subject to } x \in \Omega
\end{aligned}
$$

where $\Omega$ is a nonempty compact feasible region. Usually, there does not exist a single solution which optimizes all the objective funtions simultaneousely with cent percent satisfaction. Therefore, an appropriate method is used to generate a set of non-inferior or pareto optimal solutions from which the most preferred optimal solution(that satisfies all the objective functions with best possibility) can be determined by the decision maker (DM). 
DEFINITION [See [15]]. $x^{*} \in \Omega$ is a pareto optimal solution of MOOP (3.1) if there does not exist another feasible solution $x^{f} \in \Omega$ such that $f_{i}\left(x^{f}\right) \leq f_{i}\left(x^{*}\right)$ $\forall i$ and $f_{j}\left(x^{f}\right)<f_{j}\left(x^{*}\right)$ for at least one $j$.

\section{$4 \epsilon$-Constraint Method}

The $\epsilon$-constraint method was proposed by Haimes et al. [10] for generating pareto optimal solutions of a MOOP. It is a posteriori preference based method that generates the non-inferior solutions $[6,18]$ by considering one objective function at a time as primary one and converting the remaining objective functions as constraints. In other words, it minimizes one objective function and simultaneously maintains the maximum acceptability level for other objective functions. The $\epsilon$-constraint method is defined as:

$$
\begin{aligned}
& \operatorname{Min} f_{s}(x), s \in\{1,2, \ldots, k\} \\
& \text { subject to } \\
& f_{i}(x) \leq \epsilon_{i}, i=1,2, \ldots, s-1, s+1, \ldots, k, \quad x \in \Omega,
\end{aligned}
$$

where, $\epsilon_{i}^{L} \leq \epsilon_{i} \leq \epsilon_{i}^{U}, \epsilon_{i}^{L}=\operatorname{Min} f_{i}(x) \forall x \in \Omega, \epsilon_{i}^{U}=\operatorname{Max} f_{i}(x) \forall x \in \Omega$, i.e., the nonempty compact feasible region of the problem. Taking the value of $\epsilon_{i}$ in $\left[\epsilon_{i}^{L}, \epsilon_{i}^{U}\right]$, a sequence of optimal solutions for the objective function of high priority can be generated from which the most preferred optimal solution is determined.

\section{$5 \quad$ Fuzzy Programming}

The concept of fuzzy set theory was first proposed by Zadeh in 1965 which represents the imprecise expressions of real world problems in precise mathematical forms. Zimmermann proposed max-min operator in fuzzy approach [23] to solve multi-objective linear optimization problem. Each fuzzy set is associated with a membership function that determines the degree of membership of it's elements in the range $[0,1]$. Several types of membership functions are available in fuzzy programming to solve a multi-objective optimization problem but a suitable one is to be chosen in order to determine the optimal solution. The following steps are followed by fuzzy programming(max-min operator technique) to determine the optimal solution of an MOOP as defined in (3.1).

Step 1. Obtain the values of relative minimum $\left(L_{i}\right)$ and relative maximum $\left(U_{i}\right)$ of each objective $f_{i}(x)$ with respect to other objective functions such that $L_{i} \leq f_{i} \leq U_{i}, i=1,2, \ldots, k$.

Step 2. We consider the following membership function in our proposed method to determine the optimal solution of an MOOP. Define the fuzzy membership function $\mu(x)$ for each objective $f_{i}$ of the problem (3.1) as:

$$
\mu_{i}(x)= \begin{cases}1, & f_{i}(x) \leq L_{i} \\ \frac{U_{i}-f_{i}(x)}{U_{i}-L_{i}}, & L_{i} \leq f_{i}(x) \leq U_{i} \\ 0, & f_{i}(x) \geq U_{i}\end{cases}
$$


Step 3. Construct the following crisp model using Zimmermann's max-min operator and solve it to obtain the optimal compromise solution of MOOP (3.1).

$$
\begin{aligned}
& \operatorname{Max}\left\{\min _{1 \leq i \leq k} \mu_{i}(x)\right\} \\
& \text { subject to } x \in \Omega .
\end{aligned}
$$

This multi-objective optimization problem (5.2) is equivalent to the following problem (5.3) defined as:

$$
\begin{aligned}
& \operatorname{Max} \lambda \\
& \text { subject to } \\
& \mu_{i}(x)=\frac{U_{i}-f_{i}(x)}{U_{i}-L_{i}} \geq \lambda, i=1,2 \ldots k, \quad x \in \Omega,
\end{aligned}
$$

where $\lambda$ is an auxiliary variable and the constraints $\mu_{i}(x) \geq \lambda$ can be replaced by $f_{i}(x)+\lambda\left(U_{i}-L_{i}\right) \leq U_{i}, i=1,2, \ldots, k$ for simplicity.

Step 4. Use an appropriate method to solve the above single objective optimization problem (5.3) to obtain the optimal solution $x^{*}$ and evaluate the functional values of the objectives $f_{i}(x)(i=1,2, \ldots, k)$ at this solution to be considered as their optimal objective values.

\section{Multi-Objective LFPP with Interval Coefficients}

Multi-objective LFPP with interval coefficients of decision variables both in objective and constraint functions can be generally formulated as follows.

$$
\begin{aligned}
& \operatorname{Min} f(x)=\left(f_{1}(x), f_{2}(x), \ldots, f_{k}(x)\right) \\
& \text { subject to } \\
& \Omega=\left\{x=\left(x_{j}\right) \in \mathbb{R}^{n} \mid A x \leq b, x \geq 0\right\},
\end{aligned}
$$

where

$$
\begin{aligned}
& f_{i}(x)=\frac{\sum_{j=1}^{n} c_{i j} x_{j}+\alpha_{i}}{\sum_{j=1}^{n} d_{i j} x_{j}+\beta_{i}}, i=1,2, \ldots, k, \\
& A=\left(a_{t j}\right)_{m \times n} \text { and } b=\left(b_{t}\right) \in \mathbb{R}^{m}, t=1,2, \ldots, m .
\end{aligned}
$$

Assume that

$$
\begin{aligned}
& \sum_{j=1}^{n} d_{i j} x_{j}+\beta_{i}>0 \forall i, j \text { and } c_{i j}, d_{i j}, \alpha_{i}, \beta_{i}, a_{t j} \in I, \\
& c_{i j}=\left[c_{i j}^{L}, c_{i j}^{U}\right], d_{i j}=\left[d_{i j}^{L}, d_{i j}^{U}\right], \alpha_{i}=\left[\alpha_{i}^{L}, \alpha_{i}^{U}\right], \beta_{i}=\left[\beta_{i}^{L}, \beta_{i}^{U}\right], a_{t j}=\left[a_{t j}^{L}, a_{t j}^{U}\right]
\end{aligned}
$$

$\forall i, j, t$. Let $d_{i j}^{L}, \beta_{i}^{L}>0$ for satisfying the positivity assumption of the denominator objective functions.

The above multi-objective LFPP can be stated as follows. 
Problem $P$.

$\operatorname{Min} f_{i}(x)=\frac{\sum_{j=1}^{n}\left[c_{i j}^{L}, c_{i j}^{U}\right] x_{j}+\left[\alpha_{i}^{L}, \alpha_{i}^{U}\right]}{\sum_{j=1}^{n}\left[d_{i j}^{L}, d_{i j}^{U}\right] x_{j}+\left[\beta_{i}^{L}, \beta_{i}^{U}\right]}, i=1,2, \ldots, k$

subject to

$$
\sum_{j=1}^{n}\left[a_{t j}^{L}, a_{t j}^{U}\right] x_{j} \leq b_{t}, t=1,2, \ldots, m \text { and } x_{j} \geq 0 .
$$

As the values of $b_{t}$ mostly remains in knowledge of DM, we assume here fixed values for it but can be considered in interval form regarding the necessity of the real world problem.

The objective functions and the constraints of the above Problem $\mathrm{P}$ are formulated into the following forms.

\subsection{Objective functions}

The objective functions of Problem P can be simplified into the following forms:

$$
f_{i}(x)=\frac{\left[\sum_{j=1}^{n} c_{i j}^{L} x_{j}+\alpha_{i}^{L}, \sum_{j=1}^{n} c_{i j}^{U} x_{j}+\alpha_{i}^{U}\right]}{\left[\sum_{j=1}^{n} d_{i j}^{L} x_{j}+\beta_{i}^{L}, \sum_{j=1}^{n} d_{i j}^{U} x_{j}+\beta_{i}^{U}\right]}, i=1,2, \ldots, k .
$$

We use the following notations for convenience.

$$
f_{i}(x)=\frac{f_{i}^{N}(x)}{f_{i}^{D}(x)}=\frac{\left[f_{i}^{N L}(x), f_{i}^{N U}(x)\right]}{\left[f_{i}^{D L}(x), f_{i}^{D U}(x)\right]}, i=1,2, \ldots, k .
$$

Since $f_{i}^{D}(x)>0 \forall x \in \Omega$ (assumed), the following are the possible cases:

- Case-I: If $0 \leq f_{i}^{N L}(x) \leq f_{i}^{N U}(x)$ then $f_{i}(x)=\left[\frac{f_{i}^{N L}(x)}{f_{i}^{D U}(x)}, \frac{f_{i}^{N U}(x)}{f_{i}^{D L}(x)}\right]$.

- Case-II: If $f_{i}^{N L}(x) \leq f_{i}^{N U}(x) \leq 0$ then $f_{i}(x)=\left[\frac{f_{i}^{N L}(x)}{f_{i}^{D L}(x)}, \frac{f_{i}^{N U}(x)}{f_{i}^{D U}(x)}\right]$.

- Case-III: If $f_{i}^{N L}(x) \leq 0 \leq f_{i}^{N U}(x)$ then $f_{i}(x)=\left[\frac{f_{i}^{N L}(x)}{f_{i}^{D L}(x)}, \frac{f_{i}^{N U}(x)}{f_{i}^{D L}(x)}\right]$.

\section{Selection criterion of the cases:}

The affine functions $f_{i}^{N L}(x)$ and $f_{i}^{N U}(x)$ can be both positive and negative for different values of $x \in \Omega$. Since $f_{i}^{D}(x)>0$ (assumed), depending on the values of $f_{i}^{N L}(x)$ and $f_{i}^{N U}(x)$, the following criteria are undertaken for the problem to decide the cases that occur. As it is a minimization problem, the numerators of the objectives need to be minimized. So the signs of $f_{i}^{N L}(x)$, $f_{i}^{N U}(x)$ are considered about their individual minimal points to obtain the left and right end points of the interval form of $f_{i}(x)$. The minimum values of $f_{i}^{N L}(x)$ and $f_{i}^{N U}(x)$ are evaluated with the given constraints treating them as individual objectives and depending on the signs of their individual optimal objective values i.e., $f_{i}^{N L *}$ and $f_{i}^{N U *}$, the following observations are made.

\section{Observations:}


1. If $f_{i}^{N L *} \geq 0$ and $f_{i}^{N U *} \geq 0$ then Case-I occurs.

2. If $f_{i}^{N L *} \leq 0$ and $f_{i}^{N U *} \leq 0$ then Case-II occurs.

3. If $f_{i}^{N L *} \leq 0$ and $f_{i}^{N U *} \geq 0$ then Case-III occurs.

4. If $f_{i}^{N L *} \geq 0$ and $f_{i}^{N U *} \leq 0$ then Not Possible.

\section{Special observations:}

1. If $c_{i j}^{L}, \alpha_{i}^{L} \geq 0 \forall j=1,2, \ldots, n$ then Case-I occurs.

2. If $c_{i j}^{U}, \alpha_{i}^{U} \leq 0 \forall j=1,2, \ldots, n$ then Case-II occurs.

We consider Case-I to illustrate the procedure for computing pareto optimal solutions of the interval-valued objective functions of the MOLFPP and for the Cases-(II, III), it can be done in a similar manner.

For Case-I i.e., $\min _{x \in \Omega}\left[\frac{f_{i}^{N L}(x)}{f_{i}^{D U}(x)}, \frac{f_{i}^{N U}(x)}{f_{i}^{D L}(x)}\right]$ the optimal objective value must remain in interval form as the objective function $f_{i}(x)$ is interval-valued. However the following real-valued fractional programming problems can be considered to obtain the pareto optimal solutions for Case-I problem as we have a minimization problem.

- $\min _{x \in \Omega} \frac{f_{i}^{N L}(x)}{f_{i}^{D U}(x)}$ (Best-case problem).

- $\min _{x \in \Omega} \frac{f_{i}^{N U}(x)}{f_{i}^{D L}(x)}$ (Worst-case problem).

- $\min _{x \in \Omega} f_{i}^{N D}(x)$ where $\frac{f_{i}^{N L}(x)}{f_{i}^{D U}(x)} \leq f_{i}^{N D}(x) \leq \frac{f_{i}^{N U}(x)}{f_{i}^{D L}(x)}$ (Other-case problem).

Particularly, $\min _{x \in \Omega} \frac{f_{i}^{N L}(x)}{f_{i}^{D L}(x)}$ and $\min _{x \in \Omega} \frac{f_{i}^{N U}(x)}{f_{i}^{D U}(x)}$ belong to the class of Othercase problem, solving which the optimal objective values can be obtained in between the range of its best and worst values. Each case problem generates pareto optimal solutions but the theoretical explanation to compute the pareto optimal solutions by the proposed method is presented for the Worst-case problem.

\subsection{Constraints}

Proposition. Let $\left(x_{1}, x_{2}, \ldots, x_{n}\right)$ be a feasible point of Problem $P$ and $\lambda_{j} \in$ $\left[a_{m j}^{L}, a_{m j}^{U}\right], j=1,2, \ldots, n$ then the following results hold true.

- If $\lambda_{1} x_{1}+\lambda_{2} x_{2}+\cdots+\lambda_{n} x_{n} \leq b_{m}$ then $a_{m 1}^{L} x_{1}+a_{m 2}^{L} x_{2}+\cdots+a_{m n}^{L} x_{n} \leq b_{m}$.

- If $\lambda_{1} x_{1}+\lambda_{2} x_{2}+\cdots+\lambda_{n} x_{n} \geq b_{m}$ then $a_{m 1}^{U} x_{1}+a_{m 2}^{U} x_{2}+\cdots+a_{m n}^{U} x_{n} \geq b_{m}$.

Using the above proposition, the constraints of Problem $\mathrm{P}$ can be stated as: $\sum_{j=1}^{n} a_{t j}^{L} x_{j} \leq b_{t}, t=1,2, \ldots, m$. This reduction of constraints can be used for computing each (Best, Worst and Other)-case problm of the objective functions. If $b_{t}$ is considered as an interval $\left[b_{t}^{L}, b_{t}^{U}\right]$ then both sides of each constraint of Problem $\mathrm{P}$ exist in form of intervals. As $\left[b_{t}^{L}, b_{t}^{U}\right]$ is considered 
the range for existence of the interval $\sum_{j=1}^{n}\left[a_{t j}^{L}, a_{t j}^{U}\right] x_{j}$, the constraints can be stated as $\sum_{j=1}^{n}\left[a_{t j}^{L}, a_{t j}^{U}\right] x_{j} \subseteq\left[b_{t}^{L}, b_{t}^{U}\right]$ which can be further splitted into the following two constraints [22]:

$$
\sum_{j=1}^{n} a_{t j}^{L} x_{j} \geq b_{t}^{L} \quad \text { and } \quad \sum_{j=1}^{n} a_{t j}^{U} x_{j} \leq b_{t}^{U} .
$$

\subsection{Solution procedure by the proposed method}

Thus, the Problem $\mathrm{P}$ is transformed into Problem $\mathrm{P}^{*}$ using aforementioned objective assumptions and constraint rules.

Problem $P^{*}$.

$\operatorname{Min} f_{i}(x)=\frac{\sum_{j=1}^{n} c_{i j}^{U} x_{j}+\alpha_{i}^{U}}{\sum_{j=1}^{n} d_{i j}^{L} x_{j}+\beta_{i}^{L}}, i=1,2, \ldots, k$

subject to

$$
\sum_{j=1}^{n} a_{t j}^{L} x_{j} \leq b_{t}, t=1,2, \ldots, m \text { and } x_{j} \geq 0 .
$$

A sequence of pareto optimal solutions of Problem $\mathrm{P}^{*}$ can be generated using relative minimum and maximum of the functional values of each objective function which can be evaluated through constructing the pay-off tables by following the procedure described below.

Step 1.

$\operatorname{Min} f_{i}^{N}(x)=\sum_{j=1}^{n} c_{i j}^{U} x_{j}+\alpha_{i}^{U}$

subject to

$$
\sum_{j=1}^{n} a_{t j}^{L} x_{j} \leq b_{t}, t=1,2, \ldots, m, \quad x_{j} \geq 0 .
$$

Solve the above problem individually for each objective numerator function $f_{i}^{N}(x)$ with $i=1,2 \ldots k$. Let $X_{i}^{N *}$ be the optimal solutions of the above problem.

Step 2.

$\operatorname{Max} f_{i}^{D}(x)=\sum_{j=1}^{n} d_{i j}^{L} x_{j}+\beta_{i}^{L}$

subject to

$$
\sum_{j=1}^{n} a_{t j}^{L} x_{j} \leq b_{t}, t=1,2, \ldots, m, \quad x_{j} \geq 0 .
$$

Solve the above problem individually for each objective denominator function $f_{i}^{D}(x)$ with $i=1,2, \ldots, k$. Let $X_{i}^{D *}$ be the optimal solutions of the above problem. 
Step 3. Construct the pay-off table for objective numerator functions. Find $f_{j}^{N L}=f_{j}^{N}\left(X_{j}^{N *}\right)$ and $f_{j}^{N U}=\max \left\{f_{j}^{N}\left(X_{i}^{N *}\right) \mid i=1,2, \ldots, k\right\}$ for each objective numerator $f_{j}^{N}(x), j=1,2 \ldots k$ from the minimum and maximum values of each column of the following pay-off Table 1.

Table 1. Pay-off table for numerator functions.

\begin{tabular}{ccccc}
\hline$X_{i}^{N *}$ & $f_{1}^{N}\left(X_{i}^{N *}\right)$ & $f_{2}^{N}\left(X_{i}^{N *}\right)$ & $\ldots$ & $f_{k}^{N}\left(X_{i}^{N *}\right)$ \\
\hline$X_{1}^{N *}$ & $f_{1}^{N}\left(X_{1}^{N *}\right)$ & $f_{2}^{N}\left(X_{1}^{N *}\right)$ & $\ldots$ & $f_{k}^{N}\left(X_{1}^{N *}\right)$ \\
$X_{2}^{N *}$ & $f_{1}^{N}\left(X_{2}^{N *}\right)$ & $f_{2}^{N}\left(X_{2}^{N *}\right)$ & $\ldots$ & $f_{k}^{N}\left(X_{2}^{N *}\right)$ \\
$\vdots$ & $\vdots$ & $\vdots$ & & $\vdots$ \\
$X_{k}^{N *}$ & $f_{1}^{N}\left(X_{k}^{N *}\right)$ & $f_{2}^{N}\left(X_{k}^{N *}\right)$ & $\ldots$ & $f_{k}^{N}\left(X_{k}^{N *}\right)$ \\
\hline
\end{tabular}

Step 4. Similarly construct another pay-off table for the denominator functions to determine the relative minimum and maximum of each objective denominator $f_{j}^{D}(x), j=1,2, \ldots, k$ as:

- $f_{j}^{D L}=\min \left\{f_{j}^{D}\left(X_{i}^{D *}\right) \mid i=1,2, \ldots, k\right\}$.

- $f_{j}^{D U}=f_{j}^{D}\left(X_{j}^{D *}\right)$.

Step 5. Since $f_{i}^{N L} \leq f_{i}^{N}(x) \leq f_{i}^{N U}$ where $f_{i}^{N}(x) \leq f_{i}^{N U}$ holds true for $x=$ $X_{j}^{N *}, j=1,2, \ldots, k$ and $f_{i}^{\overline{D L}} \leq f_{i}^{D}(x) \leq f_{i}^{D U}$ where $f_{i}^{D L} \leq f_{i}^{D}(x)$ holds true for $x=X_{j}^{D *}, j=1,2, \ldots, k$, it can be considered as $f_{i}(x)=\frac{f_{i}^{N}(x)}{f_{i}^{D}(x)} \in$ $\frac{\left[f_{i}^{N L}, f_{i}^{N U}\right]}{\left[f_{i}^{D L}, f_{i}^{D U}\right]}=\left[\epsilon_{i}^{L}, \epsilon_{i}^{U}\right]$. The values of $\epsilon_{i}^{L}$ and $\epsilon_{i}^{U}$ can be determined using interval arithmetic (defined in Section 2).

For each objective function $f_{i}(x)$, the value of $\epsilon_{i}$ is restricted as $\epsilon_{i}^{L} \leq \epsilon_{i} \leq$ $\epsilon_{i}^{U}$. If $f_{s}(x)$ is highest prioritized by the DM, using $\epsilon$-constraint method, Problem $\mathrm{P}^{*}$ is transformed into the following Problem $\mathrm{P}_{s}^{*}$.

Problem $P_{s}^{*}$.

$\operatorname{Min} f_{s}(x)=\frac{\sum_{j=1}^{n} c_{s j}^{U} x_{j}+\alpha_{s}^{U}}{\sum_{j=1}^{n} d_{s j}^{L} x_{j}+\beta_{s}^{L}}, s \in\{1,2, \ldots, k\}$

subject to

$f_{l}(x) \leq \epsilon_{l}, l=1,2, \ldots, s-1, s+1, \ldots, k$ and $\Omega$.

For $f_{l}(x) \leq \epsilon_{l}, l=1,2, \ldots, s-1, s+1, \ldots, k$

$$
\frac{\sum_{j=1}^{n} c_{l j}^{U} x_{j}+\alpha_{l}^{U}}{\sum_{j=1}^{n} d_{l j}^{L} x_{j}+\beta_{l}^{L}} \leq \epsilon_{l},
$$

i.e., $\sum_{j=1}^{n}\left(c_{l j}^{U}-\epsilon_{l} d_{l j}^{L}\right) x_{j} \leq\left(\epsilon_{l} \beta_{l}^{L}-\alpha_{l}^{U}\right)$. 

form as,

Using the above results, Problem $\mathrm{P}_{s}^{*}$ can be reformulated in generalised

$\operatorname{Min} f_{s}(x)=\frac{c_{s 1}^{U} x_{1}+c_{s 2}^{U} x_{2}+\cdots+c_{s n}^{U} x_{n}+\alpha_{s}^{U}}{d_{s 1}^{L} x_{1}+d_{s 2}^{L} x_{2}+\cdots+d_{s n}^{L} x_{n}+\beta_{s}^{L}}$

subject to $\Omega$,

$$
\left(c_{l 1}^{U}-\epsilon_{l} d_{l 1}^{L}\right) x_{1}+\left(c_{l 2}^{U}-\epsilon_{l} d_{l 2}^{L}\right) x_{2}+\cdots+\left(c_{l n}^{U}-\epsilon_{l} d_{l n}^{L}\right) x_{n} \leq\left(\epsilon_{l} \beta_{l}^{L}-\alpha_{l}^{U}\right) .
$$

\section{Charnes and Cooper transformation technique}

Consider the following optimization problem with fractional objective:

$$
\text { Optimize } g\left(x_{i}\right)=\frac{\sum_{i=1}^{n} d_{i}^{\prime} x_{i}+\gamma^{\prime}}{\sum_{i=1}^{n} d_{i}^{\prime \prime} x_{i}+\gamma^{\prime \prime}} \text { subject to } \Omega \text {. }
$$

Assuming $\frac{1}{\sum_{i=1}^{n} d_{i}^{\prime \prime} x_{i}+\gamma^{\prime \prime}}=z$ and $x_{i} z=y_{i}$, the fractional objective is transformed into non-fractional linear form with an additional variable i.e.,

$$
\begin{aligned}
& \text { optimize } g\left(y_{i}, z\right)=\sum_{i=1}^{n} d_{i}^{\prime} y_{i}+\gamma^{\prime} z \\
& \text { subject to } \sum_{j=i}^{n} d_{i}^{\prime \prime} y_{i}+\gamma^{\prime \prime} z=1 \text { and } \Omega .
\end{aligned}
$$

If $\left(y_{i}^{*}, z^{*}\right)$ is the optimal solution of problem $\left(*_{2}\right)$ then $x^{*}=\left(x_{i}^{*}\right)=\left(\frac{y_{i}^{*}}{z^{*}}\right)$ is considered as the optimal solution of problem $\left(*_{1}\right)$.

Using the aforesaid transformation technique, reformulated Problem $\mathrm{P}_{s}^{*}$ can be stated as:

Problem $P_{s}^{* *}$.

$\operatorname{Min} f_{s}(y, z)=\sum_{j=1}^{n} c_{s j}^{U} y_{j}+\alpha_{s}^{U} z_{s}$

subject to

$$
\begin{aligned}
& \sum_{j=1}^{n} d_{s j}^{L} y_{j}+\beta_{s}^{L} z_{s}=1, \\
& \sum_{j=1}^{n}\left(c_{l j}^{U}-\epsilon_{l} d_{l j}^{L}\right) y_{j} \leq\left(\epsilon_{l} \beta_{l}^{L}-\alpha_{l}^{U}\right) z_{s}, l=1,2, \ldots, s-1, s+1, \ldots, k, \\
& a_{t j}^{L} y_{j} \leq b_{t} z_{s}, t=1,2, \ldots, m, \quad y_{j} \geq 0, z_{s}>0 .
\end{aligned}
$$

Changing the values of each $\epsilon_{l}$ in the interval $\left[\epsilon_{l}^{L}, \epsilon_{l}^{U}\right]$ and substituting in the constraints of the above Problem $\mathrm{P}_{s}^{* *}$, we can generate a sequence of solutions by using any suitable method. If $\left(y_{1}^{*}, y_{2}^{*}, \ldots, y_{n}^{*}, z_{s}^{*}\right)$ be the optimal solutions of the above problem then $\left(\frac{y_{1}^{*}}{z_{s}^{*}}, \frac{y_{2}^{*}}{z_{s}^{*}}, \ldots, \frac{y_{n}^{*}}{z_{s}^{*}}\right)=\left(x_{1}^{*}, x_{2}^{*}, \ldots, x_{n}^{*}\right)$ are considered as the optimal solutions of the Problem $\mathrm{P}_{s}^{*}$ and pareto optimal(non-inferior) solutions of the Problem P. Here $\epsilon$-constraint method generates $\mathrm{k}$ number of single objective LFPP i.e., Problem $\mathrm{P}_{s}^{*}$ as ' $\mathrm{s}$ ' varies in $\{1,2, \ldots, k\}$. From the sequence of pareto optimal solutions the DM can choose the most preferred optimal solution for the given multi-objective LFPP. 


\subsection{Algorithm for solving MOLFPP}

We adopt the following steps for solving MOLFPP with interval coefficients.

Step 1. Convert the given Problem $\mathrm{P}$ into Problem $\mathrm{P}^{*}$.

Step 2. Determine $\left[\epsilon_{i}^{L}, \epsilon_{i}^{U}\right]$ for each objective function $f_{i}(x)$ using the approach described in our proposed technique.

Step 3. Choose the objective function $f_{s}(x)$ of highest priority and solve Problem $\mathrm{P}_{s}^{* *}$ by changing the value of $\epsilon_{i}$ in $\left[\epsilon_{i}^{L}, \epsilon_{i}^{U}\right]$.

Step 4. Obtain a sequence of pareto optimal solutions and determine the most preferred optimal solution.

Step 5. The objective function of highest priority is altered to obtain another single objective LFPP and a new set of pareto optimal solutions can be generated using $\epsilon$-constraint method.

\section{Numerical Example}

Let us consider the following bi-objective LFPP with interval coefficients of the decision variables both in the objective and constraint functions.

Problem P.

$$
\begin{aligned}
& \operatorname{Min} f_{1}(x)=\frac{[-3,-2] x_{1}+[1,3] x_{2}+[-1,1]}{[7,8] x_{1}+[5,7] x_{2}+[8,9]}, \\
& f_{2}(x)=\frac{[1,3] x_{1}+[-3,-2] x_{2}+[1,2]}{[5,6] x_{1}+[4,7] x_{2}+[7,9]}
\end{aligned}
$$

subject to

$[2,4] x_{1}+[3,5] x_{2} \leq 6,[1,3] x_{1}+[-4,1] x_{2} \leq 3, x_{1}, x_{2} \geq 0$.

Using the selection criteria of the proposed technique (as described in Subsection 6.1), it is observed that the objective functions $f_{1}(x)$ and $f_{2}(x)$ both belong to Case-II category i.e.,

$$
f_{i}(x)=\left[\frac{f_{i}^{N L}(x)}{f_{i}^{D L}(x)}, \frac{f_{i}^{N U}(x)}{f_{i}^{D U}(x)}\right], i=1,2 .
$$

For Problem P,

$$
\begin{aligned}
& f_{1}(x)=\left[\frac{-3 x_{1}+x_{2}-1}{7 x_{1}+5 x_{2}+8}, \frac{-2 x_{1}+3 x_{2}+1}{8 x_{1}+7 x_{2}+9}\right], \\
& f_{2}(x)=\left[\frac{x_{1}-3 x_{2}+1}{5 x_{1}+4 x_{2}+7}, \frac{3 x_{1}-2 x_{2}+2}{6 x_{1}+7 x_{2}+9}\right] .
\end{aligned}
$$

So Problem $\mathrm{P}$ can be formulated into the following real model problems:

Best-case problem: $\min _{x \in \Omega}\left\{\frac{-3 x_{1}+x_{2}-1}{7 x_{1}+5 x_{2}+8}, \frac{x_{1}-3 x_{2}+1}{5 x_{1}+4 x_{2}+7}\right\}$. 
Worst-case problem: $\min _{x \in \Omega}\left\{\frac{-2 x_{1}+3 x_{2}+1}{8 x_{1}+7 x_{2}+9}, \frac{3 x_{1}-2 x_{2}+2}{6 x_{1}+7 x_{2}+9}\right\}$.

Each case can be individually solved, however we solve a case

$$
\min _{x \in \Omega}\left\{\frac{f_{1}^{N U}(x)}{f_{1}^{D L}(x)}, \frac{f_{2}^{N U}(x)}{f_{2}^{D L}(x)}\right\}
$$

in between them to generate the pareto optimal solutions i.e., Problem $\mathrm{P}^{*}$ which belongs to the category of Other-case problem as it is interpreted for Case-I category in Subsection 6.1.

Problem $P^{*}$.

$$
\begin{aligned}
\operatorname{Min} f_{1}(x) & =\frac{f_{1}^{N}(x)}{f_{1}^{D}(x)}=\frac{-2 x_{1}+3 x_{2}+1}{7 x_{1}+5 x_{2}+8}, \\
f_{2}(x) & =\frac{f_{2}^{N}(x)}{f_{2}^{D}(x)}=\frac{3 x_{1}-2 x_{2}+2}{5 x_{1}+4 x_{2}+7}
\end{aligned}
$$

subject to

$$
\Omega=\left\{\begin{array}{l}
2 x_{1}+3 x_{2} \leq 6, \\
x_{1}-4 x_{2} \leq 3, \\
x_{1}, x_{2} \geq 0
\end{array}\right.
$$

Optimizing $f_{1}^{N}(x), f_{1}^{D}(x), f_{2}^{N}(x)$ and $f_{1}^{D}(x)$ individually subject to the constraints set $\Omega$, the following results are obtained:

$$
\begin{aligned}
& \min f_{1}^{N}(x)=-5 \text { at } X_{1}^{N *}=(3,0), \\
& \min f_{2}^{N}(x)=-2 \text { at } X_{2}^{N *}=(0,2), \\
& \max f_{1}^{D}(x)=29 \text { at } X_{1}^{D *}=(3,0), \\
& \max f_{2}^{D}(x)=22 \text { at } X_{2}^{D *}=(3,0) .
\end{aligned}
$$

Constructing the pay-off table for both objective numerator and denominator functions as defined in our proposed technique, the relative minimum and maximum values of the objectives are obtained, as

$$
\begin{aligned}
& \frac{-5}{29}=\frac{f_{1}^{N L}}{f_{1}^{D U}} \leq f_{1}(x) \leq \frac{f_{1}^{N U}}{f_{1}^{D L}}=\frac{7}{29}, \\
& \frac{-2}{22}=\frac{f_{2}^{N L}}{f_{2}^{D U}} \leq f_{2}(x) \leq \frac{f_{2}^{N U}}{f_{2}^{D L}}=\frac{11}{22} .
\end{aligned}
$$

Thus, $\epsilon_{1} \in\left[\epsilon_{1}^{L}, \epsilon_{1}^{U}\right]=[-0.1724,0.2414]$ and $\epsilon_{2} \in\left[\epsilon_{2}^{L}, \epsilon_{2}^{U}\right]=[-0.0909,0.5]$.

The DM prioritizes best to either $f_{1}(x)$ or $f_{2}(x)$. Pareto optimal solutions are generated for both the cases using $\epsilon$-constraint method by mathematically modelling them as Problem $\mathrm{P}_{1}^{*}$ and Problem $\mathrm{P}_{2}^{*}$. 
Problem $P_{1}^{*}$. (The objective function $f_{1}(x)$ is prioritized best.)

$$
\operatorname{Min} f_{1}(x)=\frac{-2 x_{1}+3 x_{2}+1}{7 x_{1}+5 x_{2}+8}
$$

subject to

$$
\Omega \text { and } f_{2}(x) \leq \epsilon_{2} \text {. }
$$

Using transformation technique, it can be reformulated as:

$$
\begin{aligned}
& \operatorname{Min} f_{1}(y)=-2 y_{1}+3 y_{2}+z_{1} \\
& \text { subject to } \\
& 2 y_{1}+3 y_{2}-6 z_{1} \leq 0, \quad y_{1}-4 y_{2}-3 z_{1} \leq 0, \\
& \left(3-5 \epsilon_{2}\right) y_{1}+\left(-2-4 \epsilon_{2}\right) y_{2}-\left(7 \epsilon_{2}-2\right) z_{1} \leq 0, \\
& 7 y_{1}+5 y_{2}+8 z_{1}=1, \quad y_{1}, y_{2}, z_{1} \geq 0 .
\end{aligned}
$$

If $\left(y_{1}^{*}, y_{2}^{*}, z_{1}^{*}\right)$ be the optimal solution of the above reformulated problem then Problem $\mathrm{P}_{1}^{*}$ has optimal solution, $x_{1}^{*}=\frac{y_{1}^{*}}{z_{1}^{*}}$ and $x_{2}^{*}=\frac{y_{2}^{*}}{z_{1}^{*}}$. Now substituting different values of $\epsilon_{2}$ in reformulated form of Problem $\mathrm{P}_{1}^{*}$ such that $-0.0909 \leq$ $\epsilon_{2} \leq 0.5$, a sequence of solutions $\left(x_{1}^{*}, x_{2}^{*}\right)$ can be obtained in Table 2 which are considered as the pareto optimal solutions of Problem P.

Table 2. Solution of problem $\mathrm{P}_{1}^{*}$.

\begin{tabular}{ccccccc}
\hline$\epsilon_{2}$ & $y_{1}^{*}$ & $y_{2}^{*}$ & $z_{1}^{*}$ & $x_{1}^{*}$ & $x_{2}^{*}$ & $f_{1}^{*}=f_{1}\left(x_{1}^{*}, x_{2}^{*}\right)$ \\
\hline-0.0812 & 0.0093 & 0.1011 & 0.0537 & 0.1732 & 1.8827 & 0.3385 \\
-0.0636 & 0.0133 & 0.0968 & 0.0528 & 0.2519 & 1.8334 & 0.3168 \\
0.0069 & 0.0246 & 0.0847 & 0.0505 & 0.4871 & 1.6772 & 0.2555 \\
0.0773 & 0.0366 & 0.0718 & 0.0481 & 0.7609 & 1.4927 & 0.1903 \\
$\mathbf{0 . 1 8 3 0}$ & 0.0541 & 0.0530 & 0.0445 & $\mathbf{1 . 2 1 5 7}$ & $\mathbf{1 . 1 9 1 0}$ & $\mathbf{0 . 0 9 5 3}$ \\
0.2182 & 0.0598 & 0.0469 & 0.0434 & 1.3779 & 1.0806 & 0.0645 \\
0.2887 & 0.0711 & 0.0348 & 0.0411 & 1.7299 & 0.8467 & 0.0033 \\
0.3591 & 0.0821 & 0.0230 & 0.0388 & 2.1160 & 0.5928 & -0.0564 \\
0.3943 & 0.0875 & 0.0171 & 0.0377 & 2.3210 & 0.4536 & -0.0860 \\
0.4824 & 0.1008 & 0.0028 & 0.0350 & 2.8800 & 0.0800 & -0.1583 \\
\hline
\end{tabular}

Prioritizing best to $f_{1}(x)$ and comparing the obtained Table 2 values of $\epsilon_{2}$ and $f_{1}^{*}$, it is observed that $\left|\epsilon_{2}-f_{1}^{*}\right|$ i.e., $\left|f_{2}^{*}-f_{1}^{*}\right|$ is minimum at $x^{*}=$ $(1.2157,1.1910)$ as compared to the other solutions. Since at this solution the optimal objective values $f_{1}^{*}$ and $f_{2}^{*}$ remain in most compromised state i.e., at a shortest distance from each other, the most preferred optimal solution is considered as $x^{*}=(1.2157,1.1910)$ where the optimal objective values are $f_{1}\left(x^{*}\right)=0.0953$ and $f_{2}\left(x^{*}\right)=0.1830$. 
Problem $P_{2}^{*}$. (The objective function $f_{2}$ is prioritized best.)

$$
\operatorname{Min} f_{2}(x)=\frac{3 x_{1}-2 x_{2}+2}{5 x_{1}+4 x_{2}+7}
$$

subject to

$$
\Omega \text { and } f_{1}(x) \leq \epsilon_{1} \text {. }
$$

Using transformation technique, Problem $\mathrm{P}_{2}^{*}$ can be reformulated as:

$$
\begin{aligned}
& \operatorname{Min} f_{2}(y)=3 y_{1}-2 y_{2}+2 z_{2} \\
& \text { subject to } \\
& 2 y_{1}+3 y_{2}-6 z_{2} \leq 0, \quad y_{1}-4 y_{2}-3 z_{2} \leq 0, \\
& \left(-2-7 \epsilon_{1}\right) y_{1}+\left(3-5 \epsilon_{1}\right) y_{2}-\left(8 \epsilon_{1}-1\right) z_{2} \leq 0, \\
& 5 y_{1}+4 y_{2}+7 z_{2}=1, \quad y_{1}, y_{2} \geq 0, z_{2}>0 .
\end{aligned}
$$

If $\left(y_{1}^{*}, y_{2}^{*}, z_{2}^{*}\right)$ be the optimal solution of the above reformulated Problem $\mathrm{P}_{2}^{*}$ then $\left(x_{1}^{*}, x_{2}^{*}\right)=\left(\frac{y_{1}^{*}}{z_{2}^{*}}, \frac{y_{2}^{*}}{z_{2}^{*}}\right)$ is considered as the optimal solution of Problem $\mathrm{P}_{2}^{*}$. Now substituting different values of $\epsilon_{1}$ in the reformulated Problem $\mathrm{P}_{2}^{*}$ such that $-0.1724 \leq \epsilon_{1} \leq 0.2414$, the following pareto optimal solutions of Problem $\mathrm{P}$ can be obtained in Table-3.

Prioritizing best to $f_{2}(x)$ and comparing the values of $\epsilon_{1}$ and $f_{2}^{*}$ from the set of non-inferior solutions(in Table-3), the most preferred optimal solution can be considered as $x^{*}=(0.9948,1.3380)$ where the optimal objective values are $f_{1}\left(x^{*}\right)=0.1397$ and $f_{2}\left(x^{*}\right)=0.1332$.

Table 3. Solution of Problem $\mathrm{P}_{2}^{*}$.

\begin{tabular}{ccccccc}
\hline$\epsilon_{1}$ & $y_{1}^{*}$ & $y_{2}^{*}$ & $z_{2}^{*}$ & $x_{1}^{*}$ & $x_{2}^{*}$ & $f_{2}^{*}=f_{2}\left(x_{1}^{*}, x_{2}^{*}\right)$ \\
\hline-0.1408 & 0.1280 & 0.0082 & 0.0468 & 2.7350 & 0.1752 & 0.4610 \\
-0.1097 & 0.1198 & 0.0162 & 0.0480 & 2.4958 & 0.3375 & 0.4231 \\
-0.0473 & 0.1037 & 0.0319 & 0.0505 & 2.0535 & 0.6317 & 0.3484 \\
0.0150 & 0.0879 & 0.0473 & 0.0530 & 1.6585 & 0.8925 & 0.2752 \\
0.0773 & 0.0725 & 0.0624 & 0.0554 & 1.3087 & 1.1264 & 0.2035 \\
$\mathbf{0 . 1 3 9 7}$ & 0.0574 & 0.0772 & 0.0577 & $\mathbf{0 . 9 9 4 8}$ & $\mathbf{1 . 3 3 8 0}$ & $\mathbf{0 . 1 3 3 2}$ \\
0.2020 & 0.0426 & 0.0917 & 0.0600 & 0.7100 & 1.5283 & 0.0644 \\
0.2130 & 0.0400 & 0.0942 & 0.0604 & 0.6623 & 1.5510 & 0.0526 \\
0.2332 & 0.0353 & 0.0988 & 0.0612 & 0.5768 & 1.6144 & 0.0307 \\
0.2401 & 0.0337 & 0.1004 & 0.0614 & 0.5488 & 1.6352 & 0.0232 \\
\hline
\end{tabular}

Table-2 and Table- 3 represent the set of non-inferior or pareto optimal solutions of the given multi-objective LFPP from which the most preferred optimal solution is ascertained by the DM assigning highest priority to only one objective function and accordingly optimal objective values are determined. 


\subsection{Result by fuzzy programming}

Our example stated as Problem $\mathrm{P}$ is formulated to Problem $\mathrm{P}^{*}$ implementing some propositions. Using the pay-off tables (both for numerator and denominator objective functions) of our proposed technique, the values of relative minimum and maximum of each objective function with respect to others are ascertained as $-0.1724=L_{1} \leq f_{1}(x) \leq U_{1}=0.2414$ and $-0.0909=L_{2} \leq$ $f_{2}(x) \leq U_{2}=0.5$. According to the algorithm of max-min operator in fuzzy programming (defined in Section 5) the following crisp model obtained from Problem $\mathrm{P}^{*}$ is to be solved.

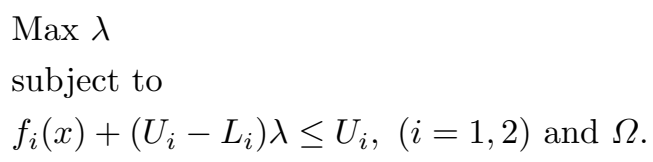

Substituting the values and on simplifying, it can be reformulated as:

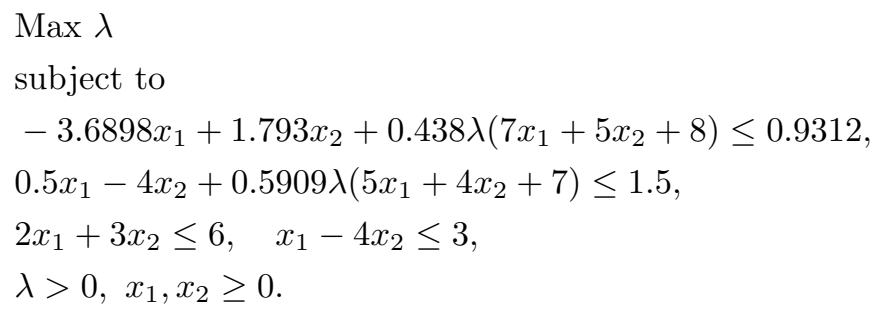

The fuzzy optimal solution is obtained as $\lambda=0.4435, x_{1}=1.4740$ and $x_{2}=$ 1.0170. Thus considering the example (converted to problem $\mathrm{P}^{*}$ ), the optimal solution obtained by fuzzy programming is $x^{*}=\left(x_{1}^{*}, x_{2}^{*}\right)=(1.4740,1.0170)$ where the optimal objective values are $f_{1}\left(x^{*}\right)=0.0471$ and $f_{2}\left(x^{*}\right)=0.2380$.

\subsection{Remarks}

In the example defined as Problem $\mathrm{P}$, our method provides the most preferred optimal solution (Table-2) by changing the values of $\epsilon_{2}$ in the constraints as $x^{*}=(1.2157,1.1910)$ and the corresponding optimal objective values are $\left(f_{1}^{*}, f_{2}^{*}\right)=(0.0953,0.1830)$. Changing the values of $\epsilon_{1}$ in the constraints, the most preferred optimal solution is considered as $x^{*}=(0.9948,1.3380)$ (Table-3) and the corresponding optimal objective values are $\left(f_{1}^{*}, f_{2}^{*}\right)=(0.1397,0.1332)$. But using fuzzy programming(max-min operator) method the optimal solution is obtained as $x^{*}=(1.4740,1.0170)$ and the corresponding optimal objective values are $\left(f_{1}^{*}, f_{2}^{*}\right)=(0.0471,0.2380)$.

Comparing the optimal objective values, it is clear that our method has several better options to choose the optimal solution and also the optimal objective values exist in more compromising state that reduces the feasibility of conflict among the objectives whereas fuzzy programming gives single optimal solution so that the DM has no choice to choose. So our method may be considered as helpful to determine the most preferred optimal solution of a MOLFPP. 


\section{Conclusions}

In this paper, $\epsilon$-constraint method is applied to generate a number of pareto optimal solutions of the multi-objective LFPP with interval coefficients both in the obective and constraint functions. As a result, several choices are available to select the most preferred optimal solution in order to satisfy all the objectives with own desired level of satisfation as all the objectives can not be fully satisfied at single solution. MATLAB programming language has been used to obtain the computational results presented in the aforementioned example. The proposed method generates set of optimal solutions which have been compared with that of fuzzy technique in the illustrated example. The pareto optimal solutions obtained by our method can be approximated with the solution due to fuzzy by adjusting the value of $\epsilon_{i}$ in the constraints. Our method gives some other better solutions than fuzzy approach so that the DM has several options to choose the best one according to own desire on preference basis. As a few methods are available in literature to solve such type of problems, our proposed method is expected to assist in this regard.

\section{Acknowledgements}

The authors are grateful to the anonymous referees and the Editor for their valuable suggestions, comments, necessary advice for the corrections to improve the quality of presentation and solution procedure of the paper. The Author (A.K. Ojha) is thankful to CSIR for the financial support of this work through the grant No:25(0201)/12/EMR-II.

\section{References}

[1] E.B. Bajalinov. Linear-Fractional Programming: Theory, Methods, Applications and Software, volume 84 of Appl. Optim. Springer, 2003.

[2] R.E. Bellman and L.A. Zadeh. Decision-making in a fuzzy environment. Management Sci., 17(4):B-141, 1970. http://dx.doi.org/10.1287/mnsc.17.4.B141.

[3] M. Borza, A.S. Rambely and M. Saraj. Solving linear fractional programming problems with interval coefficients in the objective function. A new approach. Appl. Math. Sci., 6(69):3443-3452, 2012.

[4] R. Caballero and M. Hernandez. The controlled estimation method in the multiobjective linear fractional problem. Comput. Oper. Res., 31(11):1821-1832, 2004. http://dx.doi.org/10.1016/S0305-0548(03)00141-2.

[5] A. Charnes and W.W. Cooper. Programming with linear fractional functionals. Naval Res. Logist. Q., 9(3-4):181-186, 1962. http://dx.doi.org/10.1002/nav.3800090303.

[6] Y. Collette and P. Siarry. Multiobjective Optimization: Principles and Case Studies. Springer, 2003.

[7] J.P. Costa. Computing non-dominated solutions in MOLFP. European J. Oper. Res., 181(3):1464-1475, 2007. http://dx.doi.org/10.1016/j.ejor.2005.11.051.

[8] S. Effati and M. Pakdaman. Solving the interval-valued linear fractional programming problem. Amer. J. Comput. Math., 2:51, 2012. http://dx.doi.org/10.4236/ajcm.2012.21006. 
[9] M. Ehrgott. Multicriteria Optimization. Springer, 2005.

[10] Y.Y. Haimes, L.S. Ladson and D.A. Wismer. On a bicriterion formulation of problems of integrated system identification and system optimization. IEEE Trans. Syst., Man, Cybern., Syst., 1(3):296-297, 1971. http://dx.doi.org/10.1109/TSMC.1971.4308298.

[11] M. Hladík. Generalized linear fractional programming under interval uncertainty. European J. Oper. Res., 205(1):42-46, 2010. http://dx.doi.org/10.1016/j.ejor.2010.01.018.

[12] S. Jain and N. Arya. Inverse optimization for linear fractional programming. Int. J. Phys. Math. Sci., 4(1), 2013.

[13] M.K. Luhandjula. Fuzzy approaches for multiple objective linear fractional optimization. Fuzzy Sets and Systems, 13(1):11-23, 1984. http://dx.doi.org/10.1016/0165-0114(84)90023-X.

[14] B. Martos. Hyperbolic programming. Publ. Res. Inst. Math. Sci., 5:386-407, 1960 .

[15] K.M. Miettinen. Nonlinear Multiobjective Optimization. Kluwer Academic Publisher, 2004.

[16] R.E. Moore. Interval Analysis. Prentice-Hall Englewood Cliffs, 1966.

[17] I. Nykowski and Z. Zolkiewski. A compromise procedure for the multiple objective linear fractional programming problem. European J. Oper. Res., 19(1):9197, 1985. http://dx.doi.org/10.1016/0377-2217(85)90312-1.

[18] A.K. Ojha and K.K. Biswal. Multi-objective geometric programming problem with $\epsilon$-constraint method. Appl. Math. Model., 38(2):747-758, 2014. http://dx.doi.org/10.1016/j.apm.2013.07.003.

[19] B.B. Pal and S. Sen. A goal programming procedure for solving interval valued multiobjective fractional programming problems. In Advanced Computing and Communications, 2008. ADCOM 2008. 16th International Conference on, pp. 297-302. IEEE, 2008.

[20] I.M. Stancu-Minasian. Fractional Programming. Springer, 1997.

[21] H. Suprajitno. Solving multiobjective linear programming problem using interval arithmetic. Appl. Math. Sci., 6(80):3959-3968, 2012.

[22] H.C. Wu. On interval-valued nonlinear programming problems. J. Math. Anal. Appl., 338(1):299-316, 2008. http://dx.doi.org/10.1016/j.jmaa.2007.05.023.

[23] H.-J. Zimmermann. Fuzzy programming and linear programming with several objective functions. Fuzzy Sets and Systems, 1(1):45-55, 1978.

http://dx.doi.org/10.1016/0165-0114(78)90031-3. 